\title{
Acquisition of new tumor cell properties by MSC-derived exosomes
}

\author{
YUANYUAN YANG ${ }^{1,5}$, VESNA BUCAN ${ }^{2}$, HEIKE BAEHRE ${ }^{3,4}$, \\ JULIANE VON DER OHE ${ }^{1}$, ANNA OTTE ${ }^{1}$ and RALF HASS ${ }^{1}$ \\ ${ }^{1}$ Biochemistry and Tumor Biology Laboratory, Department of Obstetrics and Gynecology, \\ ${ }^{2}$ Department of Plastic, Hand and Reconstructive Surgery, ${ }^{3}$ Institute of Pharmacology, \\ ${ }^{4}$ Research Core Unit Metabolomics, Hannover Medical School, Hannover, Germany; \\ ${ }^{5}$ Tongji Hospital Affiliated to Tongji University, Shanghai 200065, P.R. China
}

Received March 6, 2015; Accepted April 9, 2015

DOI: $10.3892 /$ ijo.2015.3001

\begin{abstract}
Interaction between multi-functional mesenchymal stroma/stem cells (MSC) and human tumor cells involves the exchange of biological material via extracellular vesicles including exosomes. Protein analysis of MSC-derived exosomes demonstrated the presence of MMP-2 and MSC-specific markers including CD90 and ecto-5'-nucleotidase (CD73). Incubation of tumor cells with these membranous particles revealed a rapid uptake of MSC-released microvesicles whereby breast cancer cells incorporated $\sim 19 \%$ and SCCOHT-1 cells representing a rare type of small cell ovarian cancer assimilated $\sim 28 \%$ of available exosomes within $24 \mathrm{~h}$. This interaction was accompanied by functional alterations of tumor cell properties during integration of exosomal content from MSC. Indeed, exosome-associated MMP-2 exhibited functional enzyme activity and MCF-7 breast cancer cells with undetectable MMP-2 protein acquired expression of this enzyme and corresponding gelatinase functionality after stimulation with MSC-derived exosomes. Similar effects were observed in SCCOHT-1 cells during culture in the presence of MSC-derived exosomes which enabled new metabolic activities in this tumor cell type. Together, these findings demonstrated that the internalization of MSC-derived exosomes was associated with the acquisition of new tumor cell properties by altering cellular functionalities and providing the capability to re-organize the tumor microenvironment.
\end{abstract}

\section{Introduction}

Multipotent human mesenchymal stroma/stem cells (MSC) are characterized as a heterogeneous cell population which can be

Correspondence to: Professor Ralf Hass, Biochemistry and Tumor Biology Laboratory, Department of Gynecology and Obstetrics, Medical University Hannover, Carl-Neuberg-Str. 1, D-30625 Hannover, Germany

E-mail: hass.ralf@mh-hannover.de

Key words: mesenchymal stem cells, SCCOHT-1, breast cancer, tumor cell signaling, intercellular communication, exosomes found in nearly all vascularized organs and tissues. The stem cell properties include self-renewal and regenerative potential (1). Moreover, MSC display at least a tri-lineage differentiation capacity along the osteogenic, chondrogenic and adipogenic phenotype (2). Certain heterogeneity in morphology and cell fate as demonstrated by isolation of MSC subpopulations (3) may result partially from the cellular microenvironment by neighboring cells, altered trophic factors, $\mathrm{pH}$ or hypoxic conditions. Consequently, a broad range of simultaneous properties including the capacity for plastic adherence, paralleled by expression of the CD73, CD90 and CD105 surface molecules with concomitant absence of other cell type-specific markers including CD14, CD31, CD34 CD45 and HLA-DR can be identified for MSC $(2,4)$.

MSC are recruited during tissue damage to support wound repair and tissue regeneration. Likewise, invasive tumor growth also causes tissue injuries and inflammatory processes with the consequence of MSC attraction and cellular crosstalk. MSC can interact with tumor cells via exchange of soluble factors like chemokines, cytokines and further trophic molecules as well as various microvesicles including exosomes. These types of interaction enable multiple pathways for MSC to communicate with neighboring tumor cells whereby release of exosomes can also affect more distant tumor cells. Exosomes represent small membrane particles of endocytic origin which are released into the extracellular compartment (5) and contain a large panel of proteins, mRNAs and regulatory microRNAs (miRs) which can alter the functionality of recipient cells (6). According to the heterogeneity of MSC populations, exosomes from MSC of different tissue origin contain a variety of unique proteins together with some common exosomal marker proteins such as CD29 or CD63 $(7,8)$. Several effects of MSC-derived exosomes on tumor cells have been demonstrated including suppression of angiogenic potential by down-modulation of VEGF in breast cancer cells via exosome-associated miR-16 (9). Moreover, human umbilical cord MSC-derived exosomes can protect against cisplatin-induced nephrotoxicity and promote cell proliferation (10) and other research has demonstrated that human bone marrow MSC-derived exosomes increase tumor growth in vivo (11), however, mechanisms for these findings remain unclear. 
In the present study, we investigated the effects of MSC-derived exosomes on different tumor types including breast cancer and a rare type of ovarian carcinoma cells. The data demonstrated a variety of functional changes including MMP-2 and ecto-5'-nucleotidase acquisition by different tumor cells following internalization of exosomes.

\section{Materials and methods}

Cell culture of mesenchymal stem/stroma cells (MSC). Primary human mesenchymal stem cells were obtained after explant culture of umbilical cord tissue; the procedure was approved by the Ethics Committee of Hannover Medical School, Project no. 443, February 26, 2009, respectively, following informed written consent by the patient.

MSC-like cells were isolated from human umbilical cords as reported previously (12). The cells were obtained from different patients following delivery of full-term (38-40 weeks) infants either spontaneously or by cesarean section. In brief, umbilical cord tissue was washed with PBS to remove blood cells, cut into $\sim 0.5 \mathrm{~cm}^{3}$ large pieces and incubated in $\alpha \mathrm{MEM}$ (Sigma Chemie GmbH, Steinheim, Germany) supplemented with $15 \%$ of allogeneic human AB-serum (HS; commercially obtained from blood bank, University Campus Lübeck, Germany), $100 \mathrm{U} / \mathrm{ml}$ penicillin, $100 \mu \mathrm{g} / \mathrm{ml}$ streptomycin and $2 \mathrm{mM}$ L-glutamine (Sigma) at $37^{\circ} \mathrm{C}$ in a humidified atmosphere with $5 \% \mathrm{CO}_{2}$. After $\sim 14$ days of explant culture, the umbilical cord tissue pieces were removed and the adherent cells were harvested by accutase (Sigma) treatment for $3 \mathrm{~min}$ at $37^{\circ} \mathrm{C}$. The obtained cell suspension was centrifuged at $320 \mathrm{x}$ g for 5 min and the cells were resuspended in MSC culture medium ( $\alpha$ MEM supplemented with $10 \% \mathrm{HS}, 100 \mathrm{U} / \mathrm{ml}$ penicillin, $100 \mu \mathrm{g} / \mathrm{ml}$ streptomycin and $2 \mathrm{mM}$ L-glutamine) and subcultured in the appropriate passage. For the experiments, MSC primary cultures from 5 different donors in different passages (P1 to P5) were used (MSC241111 in P3, MSC131113 in P2, MSC101213 in P1 and P3, MSC180314 in P5 and MSC270114 in $\mathrm{P} 1)$, respectively.

Cell culture of tumor cells. Human MDA-MB-231 and MCF-7 breast carcinoma cell lines were obtained from the American Type Culture Collection (Rockville, MD, USA). MCF-7 cells were grown in Dulbecco's modified Eagle's medium and MDA-MB-231 cells were cultured in Leibovitz medium supplemented with $[10 \%$ (v/v) fetal calf serum, $2 \mathrm{mM}$ L-glutamine, $100 \mathrm{U} / \mathrm{ml}$ penicillin and $100 \mathrm{mg} / \mathrm{ml}$ streptomycin] (all from Sigma Chemie GmbH, Steinheim, Germany), respectively. Subculture was performed by trypsin/EDTA (Biochrom $\mathrm{GmbH}$, Berlin, Germany) treatment for $5 \mathrm{~min}$ at $37^{\circ} \mathrm{C}$.

SCCOHT-1 cells represent a spontaneously proliferating population derived from a patient with recurrent small cell carcinoma of the ovary hypercalcemic type (SCCOHT) and were maintained in RPMI-1640 with medium supplements as described previously (13).

Cells were cultivated at $37^{\circ} \mathrm{C}$ in a humidified atmosphere containing $5 \% \mathrm{CO}_{2}$ and tested for mycoplasma by the luminometric MycoAlert Plus mycoplasma detection kit (Lonza Inc., Rockland, ME, USA) according to the manufacturer's recommendations. Moreover, authentication of the cell lines was performed by short tandem repeat (STR) fragment analysis using the GenomeLab human STR primer set (Beckman Coulter Inc., Fullerton, CA, USA) demonstrating similar STR pattern according to the STR database provided by the Deutsche Sammlung von Mikroorganismen und Zellkulturen (DSMZ, Braunschweig, Germany).

Labeling of MSC by lentiviral transduction. For discrimination of the different tumor cells in co-culture with MSC and for proliferation measurements, all tumor cell populations were transduced with a 3rd generation lentiviral SIN vector containing the mcherry gene. Likewise, the different MSC populations were transduced with a 3 rd generation lentiviral SIN vector containing the eGFP gene according to a labeling technique previously described (14).

Analysis of surface markers byflow cytometry. Characterization of the MSC immunophenotype was performed as described previously (15). Briefly, continuously proliferating MSC were harvested and analyzed for cell surface marker expression by flow cytometry. After blocking non-specific binding to Fc-receptors by incubation of $10^{6}$ cells with $2 \%$ FCS for $30 \mathrm{~min}$ at $4^{\circ} \mathrm{C}$ and washing with PBS-BSA, the cells were incubated with the following appropriately-labeled monoclonal anti-human antibodies, respectively: CD73-PE (clone AD2) (BD Bioscience); CD90-PE (clone 5E10, IgG1, BioLegend Inc., San Diego, CA, USA); CD105-PE (clone 43A3, IgG1, BioLegend Inc.); CD31-FITC (Miltenyi Biotec, BergischGladbach, Germany); CD34-PE and CD45-PE(BD Biosciences). Following antibody staining, all samples were washed twice with PBS-BSA. Positive staining was obtained according to control measurements of the different populations with isotype-matching IgG control antibodies. Flow cytometry analysis and histograms were performed in a Galaxy FACSan (Partec) using FloMax analysis software (Partec).

Preparation of exosomes. Exosomes were isolated using the total exosome isolation kit reagent (Invitrogen, USA). After culture in serum-free conditions for $24 \mathrm{~h}$, cell media were harvested from MSC ${ }^{\mathrm{GFP}}, \mathrm{MCF}-7^{\text {cherry }}$, and MDA-MB231 $1^{\text {cherry }}$ mono-cultures and from co-cultures of MSC with the two breast cancer cell lines, respectively. Co-culture of MSC and the tumor cells was performed at a cell ratio of $60: 40$. The cells were cultured at an initial density of 2,000 cells $/ \mathrm{cm}^{2}$ for 7-15 days and following medium exchange with serum-free media, the released exosomes were isolated after subsequent $24 \mathrm{~h}$.

The serum-free cell media samples were centrifuged at $2,000 \mathrm{x} \mathrm{g}$ for $30 \mathrm{~min}$ to remove cell debris. The supernatant was stored on ice and supplemented with the half volume of the total exosome isolation kit reagent (Invitrogen) according to the manufacturer's instructions. After thorough mixing, the samples were incubated at $4^{\circ} \mathrm{C}$ overnight and centrifuged at $100,000 \times \mathrm{g} / 4^{\circ} \mathrm{C}$ for $1 \mathrm{~h}$. The supernatant was aspirated and discarded, and the exosome pellet was resuspended for protein and enzymatic analyses.

For incorporation of $\mathrm{MSC}^{\mathrm{GFP}}$-derived exosomes into tumor cells, equal aliquots of exosomes were added to $10^{4}$ MCF-7 or SCCOHT-1 cells in a 6-well plate (Nunc). The tumor cell cultures were then incubated for $24 \mathrm{~h}$ in the presence of $\mathrm{MSC}^{\mathrm{GFP}}$-derived exosomes followed by 5 extensive 
washes of the wells with PBS to remove free and loosely cell-attached exosomes. Thereafter, the cells were detached by trypsin/EDTA treatment, homogenized in appropriate buffer and cell homogenates were analyzed by western blot analysis or zymography assay. Alternatively, analysis of exosomes and of the tumor cells with incorporated GFP-labeled exosomes was performed after lysis in $10 \%(\mathrm{w} / \mathrm{v})$ SDS. Relative fluorescence intensities of the homogenates were quantified for GFP (excitation $485 \mathrm{~nm} /$ emission $520 \mathrm{~nm}$ ) using the Fluoroscan Ascent Fl (Thermo Fisher Scientific).

$2 D$-gel analysis and mass spectrometry identification. The proteins of the different exosome preparations were separated by isoelectric focusing (IEF) followed by SDS-PAGE in the second dimension, respectively. Thus, aliquots of exosomes from each preparation were incubated in reswelling buffer [8 M urea, 1\% CHAPS (v/v), 0.5\% pharmalytes 3-10 (v/v), $0.002 \%$ bromophenol blue (w/v), $0.4 \%$ DTT (w/v); according to the GE Healthcare protocol] on an 18-cm IPG Immobiline Dry Strip (pH 3.0-10.0; NL) (Amersham Biosciences GmbH, Freiburg, Germany) and separated for $18 \mathrm{~h}$ at $150 \mathrm{~V}$ in the first dimension using the IPGphor isoelectric focusing system (Amersham). Thereafter, the IPG strips were incubated in two subsequent equilibration buffers for $15 \mathrm{~min}$, respectively (according to the GE Healthcare protocol), and polymerized on a $10 \%$ SDS-PAGE separation gel using $0.5 \%(\mathrm{w} / \mathrm{v})$ low melting point agarose. Electrophoresis was standardized using appropriate molecular-weight markers (Amersham). Following staining of the gels with Coomassie brilliant blue appropriate protein spots were cut and analyzed by liquid chromatography coupled with tandem mass spectrometry (LC-MS/ MS) using the AB5800 TOF/TOF (ABSys GmbH, Darmstadt, Germany).

Western blot analysis. Exosome aliquots from the different cell cultures were homogenized in RIPA buffer containing $0.3 \mathrm{M} \mathrm{NaCl}, 1 \%(\mathrm{w} / \mathrm{v})$ sodium desoxycholate, $0.1 \%(\mathrm{w} / \mathrm{v})$ sodium dodecyl sulfate (SDS), $1 \%$ (v/v) Triton X-100, $20 \mathrm{mM}$ Tris- $\mathrm{HCl}$ (pH 8.0), $1 \mathrm{mM}$ EDTA supplemented with $1 \mathrm{mM}$ phenylmethylsulfonylfluoride (PMSF). Approximately $15 \mu \mathrm{g}$ of exosomal protein was separated by electrophoresis on a $15 \%$ SDS-polyacrylamide gel and transferred to a PVDF membrane (Millipore Corp., Bedford, MA, USA). Immunoblotting was performed with the following antibodies: polyclonal anti-CD63; polyclonal anti-collagen $\alpha \mathrm{I}$; polyclonal anti-collagen $\alpha$ II, polyclonal anti-CD73 and antiMMP2 (each 1:500 dilution; all from Abcam, Cambrige, UK); monoclonal anti-CD90 (1:500 dilution; Dianova, Hamburg, Germany). Odyssey 680/800 $\mathrm{nm}$ secondary conjugates were used for the quantification of protein expression levels and signals were visualized using the Odyssey Infra-Red Imaging System and software (Li-Cor BioSciences, Lincoln, NE, USA).

Transcript analysis by RT-PCR. Total RNA was isolated from MSC and MSC-derived exosomes using RNeasy Mini kit (Qiagen, Hilden, Germany) according to the manufacturer's instructions. One microgram of RNA was reverse transcribed into cDNA using $500 \mu \mathrm{M}$ of dNTP (R0193), $5 \mu \mathrm{M}$ Oligo(dT)18 primer (S0132), $5 \mu \mathrm{M}$ Random Hexan primer (S0142), $1 \mathrm{U}$
RiboLock $^{\mathrm{TM}}$ RNase inhibitor (E00381) and $5 \mathrm{U}^{\text {RevertAid }}{ }^{\mathrm{TM}}$ M-MuLV reverse transcriptase (EP0441) in the supplied reaction buffer (all reagents from Thermo Scientific, Schwerte, Germany). The cDNA reactions were performed for $10 \mathrm{~min} / 25^{\circ} \mathrm{C}$, $1 \mathrm{~h} / 37^{\circ} \mathrm{C}$ and stopped at $72^{\circ} \mathrm{C}$ for $10 \mathrm{~min}$. As a template $2.5 \mu \mathrm{l}$ of cDNA was used with primers specific for: CD73 (forward, 5'-CGCAACAATGGCACAATTAC-3'; reverse, 5'-CTCGACA CTTGGTGCAAAGA-3'; amplification product $241 \mathrm{bp}$ ); CD90 (forward, 5'-GGACTGAGATCCCAGAACCA-3'; reverse, 5'-ACGAAGGCTCTGGTCCACTA-3'; amplification product 124 bp); CD105 (forward, 5'-TGTCTCACTTCATGCCTCC AGCT-3'; reverse, 5'-AGGCTGTCCATGTTGAGGCAGT-3'; amplification product $378 \mathrm{bp}$ ); MMP-2 (forward, 5'-TTTTCT CGAATCCATGATGG-3'; reverse, 5'-CTGGTGCAGCTCT CATATTT-3'; amplification product $619 \mathrm{bp}$ ); fibronectin (forward, 5'-AGCCGCCACGTGCCAGGATTAC-3'; reverse, 5'-CTTATGGGGGTGGCCGTTGTGG-3'; amplification product $439 \mathrm{bp}$ ); (all primers customized by Eurofins, MWG $\mathrm{GmbH}$, Ebersberg, Germany). PCR reactions included $0.2 \mu \mathrm{M}$ of each primer, $200 \mu \mathrm{M}$ of dNTP (R0193, Thermo Scientific) and 0.03 U One Taq Hot Start DNA polymerase (New England Biolabs GmbH, Frankfurt am Main, Germany) in the supplied reaction buffer. PCR cycling conditions were performed $30 \mathrm{sec}$ at $94^{\circ} \mathrm{C}, 1 \mathrm{~min}$ at 60 and $68^{\circ} \mathrm{C}$ for $1 \mathrm{~min}$, respectively, including an initial 30 -sec denaturation step at $94^{\circ} \mathrm{C}$ and a final 5-min extension step at $68^{\circ} \mathrm{C}$ ( 35 cycles). Aliquots of $25 \mu \mathrm{l}$ of each RT-PCR product were separated on a $2 \%$ agarose gel and visualized by GelRed $^{\mathrm{TM}}$ (Biotium Inc., Hayward, CA, USA) staining.

MMP-2 zymography assay. Exosome aliquots from the MSC ${ }^{\mathrm{GFP}}$, MDA-MB-231 $1^{\text {cherry }}$ cells, and from co-cultures of MSC ${ }^{G F P /}$ MDA-MB-231 $1^{\text {cherry }}$ cells were used in a zymographic assay. Moreover, conditioned media was prepared from $10^{6} \mathrm{MSC}^{\mathrm{GFP}}$ or MCF- $7^{\text {cherry }}$ cells or MCF- $7^{\text {cherry }}$ cells with incorporated MSC $^{\text {GFP }}$-derived exosomes after 24 -h culture in $0.1 \%$ serum and concentrated 20 -fold using Amicon Ultra-4 Centrifugal Filter Devices (10 kDa; Millipore, Carrigtwohill, Ireland) according to the manufacturer's instructions. In the following MMP-2 zymographic assay, aliquots were mixed 2:1 (v/v) with non-reducing sample buffer [10 mM Tris (pH 6.0-8.0), $1 \%$ SDS, $10 \%$ glycerol and $0.02 \%$ bromophenol blue] and subjected to SDS-PAGE containing $2 \mathrm{mg} / \mathrm{ml}$ of gelatine (Sigma). Electrophoresis was performed for $30 \mathrm{~min}$ at $60 \mathrm{~V}$ followed by $120 \mathrm{~min}$ at $125 \mathrm{~V}$. The gels were washed twice in $2.5 \%$ Triton $\mathrm{X}-100$ on a vertical shaker and five times with $\mathrm{H}_{2} \mathrm{O}$. Thereafter, the gels were incubated with fresh MMP enzyme buffer ( $50 \mathrm{mM}$ Tris-HCl, $\mathrm{pH} 7.0$, $5 \mathrm{mM} \mathrm{CaCl}_{2}$ ) overnight at $37^{\circ} \mathrm{C}$. Finally, the gels were stained with $0.4 \%$ Coomassie blue whereby the proteolytic activity was detectable by the appearance of light bands.

CD73 activity by analysis of 5'-AMP and adenosine. Exosomes were isolated from steady-state SCCOHT-1 mono-cultures after 7 days, from steady-state MSC101213 P3 mono-cultures after 7 days, and from 7-day co-culture of these MSC with SCCOHT-1 (ratio 60:40; initial seeding of 2,000 cells $/ \mathrm{cm}^{2}$ ). The appropriate exosomal fractions as well as SCCOHT-1 control cells and SCCOHT-1 cells with $24 \mathrm{~h}$ incorporated MSC-derived exosomes were cultivated in PBS with $20 \mu \mathrm{M}$ 5'-AMP (Sigma, Schnelldorf, Germany) as a substrate for 


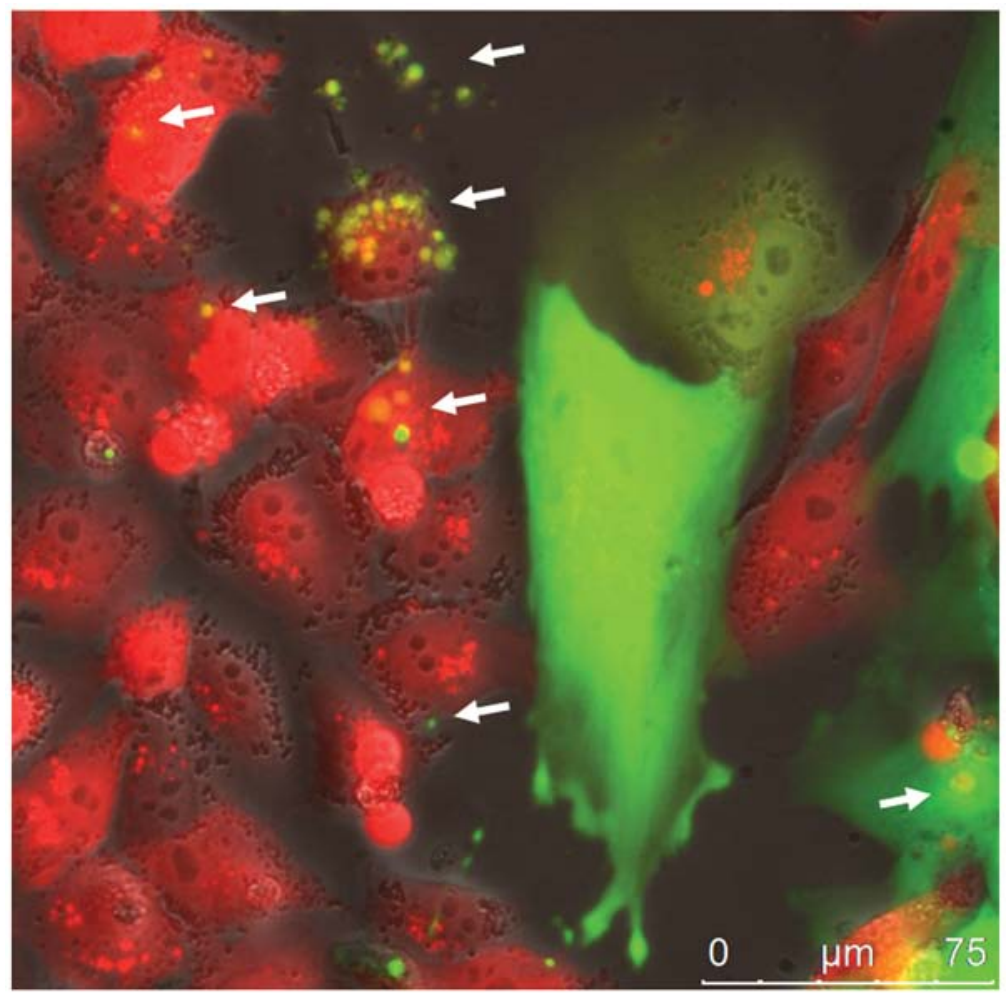

Figure 1. Exchange of extracellular vesicles between MSC and tumor cells. MSC101213 ${ }^{\text {GFP }}$ P1 and MDA-MB-231 ${ }^{\text {cherry }}$ breast cancer cells demonstrated the release of green fluorescing exosomes from MSC into the extracellular compartment and a corresponding uptake of these MSC-derived vesicles by various breast cancer cells in 7-day co-culture (white arrows). Bar, $75 \mu \mathrm{m}$.

30 min at $37^{\circ} \mathrm{C}$. An exosome-free and a cell-free PBS incubation served as a negative control, respectively. Supernatants were collected and centrifuged $(14.000 \mathrm{x} \mathrm{g} / 5 \mathrm{~min})$ to remove debris and supernatants were analyzed by HPLC-MS/MS using a Shimadzu HPLC-system (Shimadzu, Duisburg, Germany) coupled with a QTRAP5500 ${ }^{\mathrm{TM}}$ triple quadrupole mass spectrometer (ABSCIEX, Foster City, CA, USA) operating in positive ionization mode. Reversed phase chromatographic separation of adenosine and 5'-AMP was performed on a Hypercarb column (30x4.6 mm; $5 \mu \mathrm{m}$; Thermo Scientific, Dreieich, Germany) using a linear organic gradient. Data were collected and analyzed with Analyst 1.5.1 software (ABSCIEX).

\section{Results}

Direct cellular interaction of MSC ${ }^{\text {GFP }}$ with MDA-MB-231 $1^{\text {cherry }}$ breast cancer cells was associated with the exchange of exosomelike micovesicles (Fig. 1). GFP-labeled exosomes derived from MSC241111 ${ }^{\text {GFP }} \mathrm{P} 3$ were detectable within the extracellular space and were incorporated into MDA-MB-231 $1^{\text {cherry }}$ breast cancer cells as indicated by the white arrows (Fig. 1). While these cellular interactions and the exchange of exosomes suggested an intercellular transfer of biological material between MSC and the tumor cells, a 24-h production of exosomes was isolated from the mono-cultures and the co-cultures for further analysis. The protein amount of isolated exosomes from MDA-MB-231 $1^{\text {cherry }}$ mono-cultures was $108 \mu \mathrm{g}$, from MSC241111 ${ }^{\text {GFP }}$ was $90 \mu \mathrm{g}$, and the co-culture of MSC ${ }^{\text {GFP }}$ with MDA-MB-231 ${ }^{\text {cherry }}$ yielded $144 \mu \mathrm{g}$ within $24 \mathrm{~h}$. Comparative protein analysis was performed by 2D-gel separation of $35 \mu \mathrm{g}$ of the exosomal protein fraction of each preparation (Fig. 2). A variety of common protein spots were detectable in all 3 preparations, however, at least two additional proteins were identified in MSC exosomes (Fig. 2, upper panel) and co-culture exosomes (Fig. 2, lower panel) in contrast to MDA-MB-231 exosomes (Fig. 2, middle panel). Mass spectrometric analysis revealed fibronectin and matrix metalloproteinase-2 (MMP-2) as those protein spots which were undetectable in exosomes from MDA-MB-231 cells (Fig. 2). Western blot analysis of exosomal preparation confirmed these findings (Fig. 3A). Moreover, similar data were also obtained from exosomes after co-culture of MSC with MCF-7 breast cancer cells (Fig. 3B). In addition to the presence of MMP-2 exclusively in exosomes from MSC and the co-culture, the GPI-anchored CD90 antigen was similarly detectable and likewise the ecto-5'-nucleotidase CD73, all of which remain absent in the two breast cancer cell lines (Fig. 3). Whereas fibronectin can associate with certain types of collagen, the isoform collagen $\alpha 1$ was also detectable in exosomes from MSC mono-cultures and was reduced in the co-culture exosomes, however, it was undetectable in the exosome preparation of MDA-MB-231 and MCF-7 cells, respectively (Fig. 3). In contrast, collagen $\alpha 2$ appeared in all exosome preparations and likewise, CD63 as an exosomal marker protein was uniformly present (Fig. 3).

Furthermore, transcripts of the typical MSC marker proteins CD73, CD90, and CD105 were detectable in MSC lysates as well as MMP2 and fibronectin in accordance with the mass spectrometric analysis of the 2D gels (Fig. 4). Likewise, mRNAs of CD73, CD90, and fibronectin also 


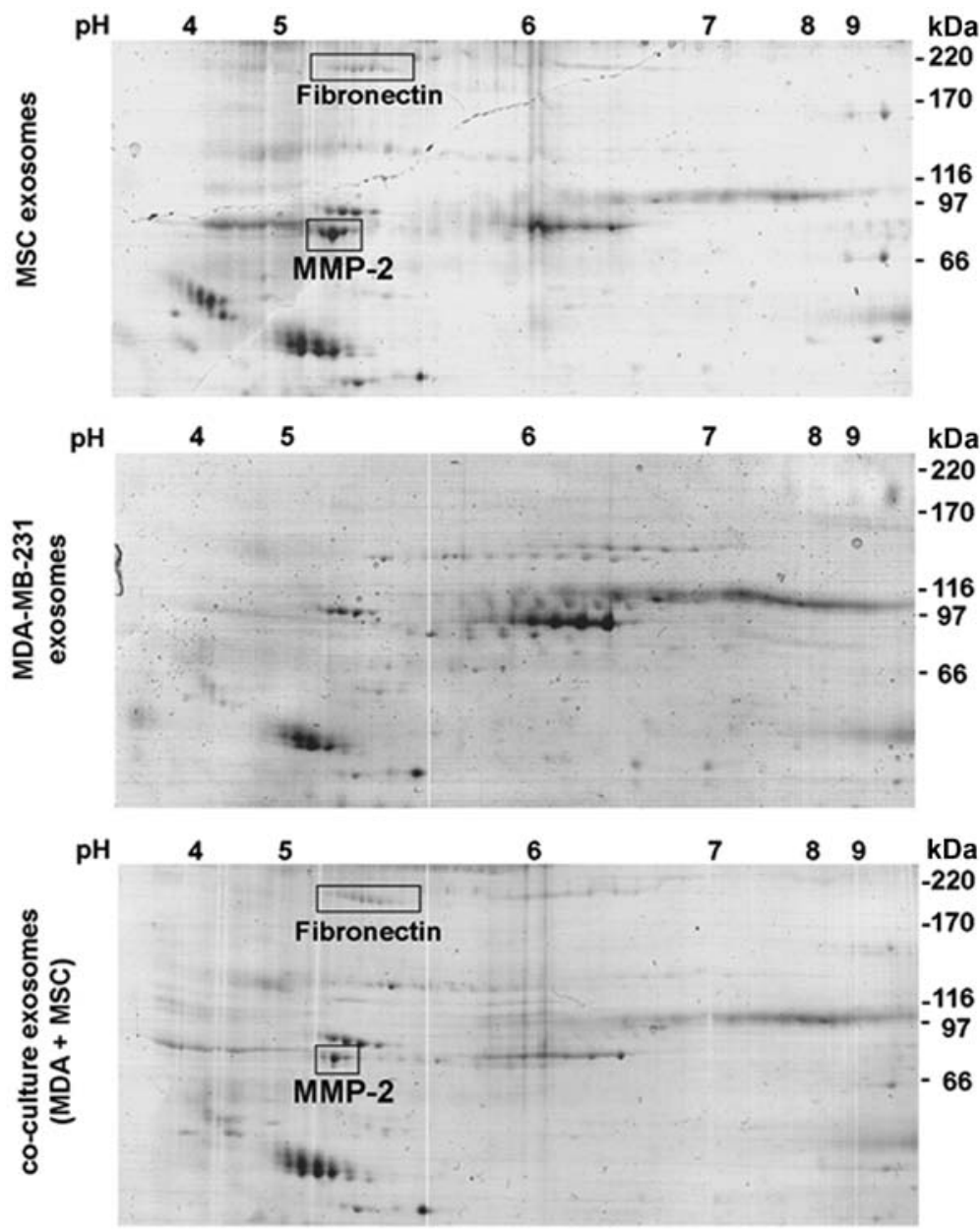

Figure 2. Two dimensional (2D)-gel analysis of exosomal proteins was performed from mono-cultures of MSC241111 P3 (upper panel), MDA-MB-231 cells (middle panel), and during co-culture of MSC241111 P3 with MDA-MB-231 cells (lower panel) by applying $35 \mu \mathrm{g}$ of exosomal protein to the gels, respectively, whereas exosomes from MSC mono- and co-cultures demonstrated a similar protein pattern, differences compared to proteins derived from the breast cancer cell exosomes were analyzed by liquid chromatography coupled with tandem mass spectrometry and revealed fibronectin and MMP- 2 indicated on the upper and lower panel, respectively.

A

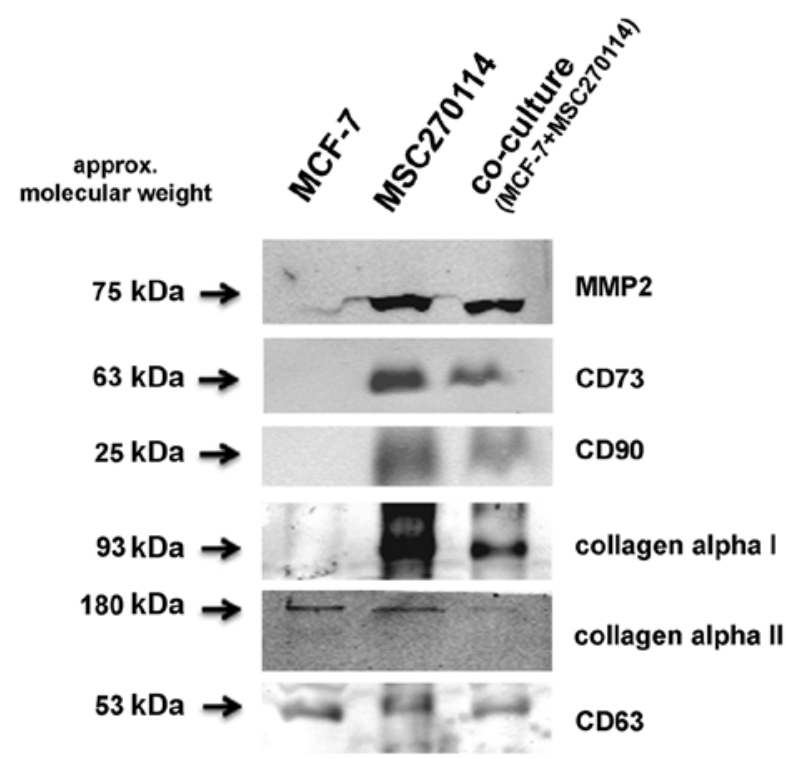

B

\section{Exosomes}

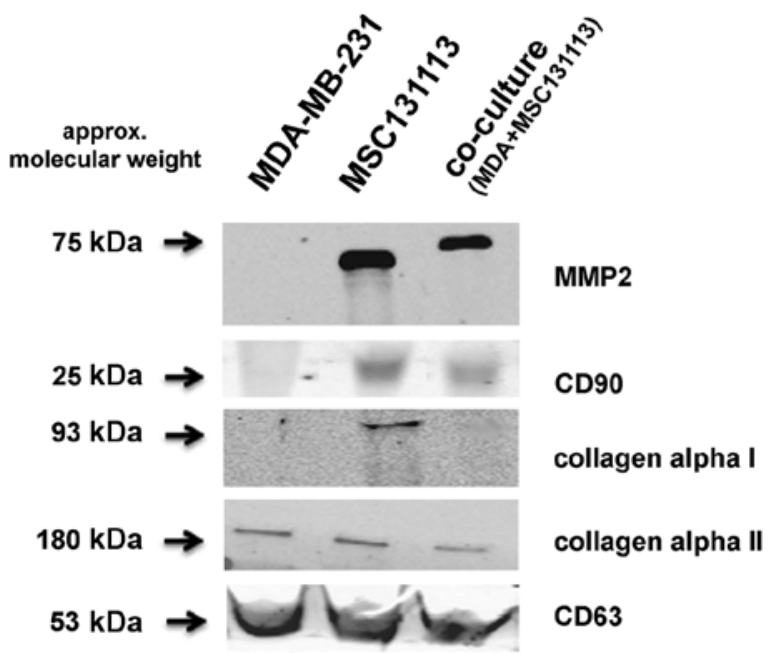

Figure 3. Western blot analysis was performed with $15 \mu \mathrm{g}$ of exosomal proteins from mono-cultures of MCF-7 cells and MSC270114 P1 as well as their coculture (A), respectively, and likewise from mono-cultures of MDA-MB-231 cells and MSC131113 P2 with their corresponding co-culture (B), respectively. The unaltered expression of CD63 as exosomal marker protein was used as a control. 
$\mathrm{MSC}^{\mathrm{GFP}}$
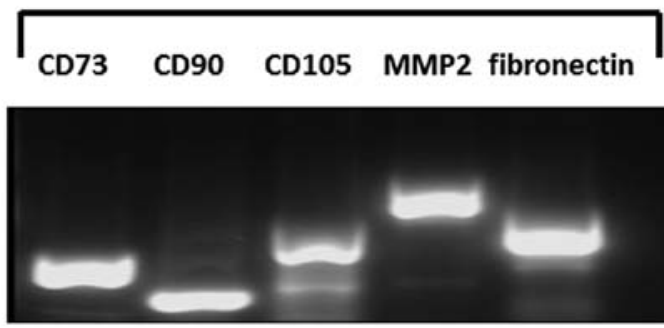

bp 241
124

378

619

\section{MSC GFP-derived exosomes}

\section{CD73 CD90 CD105 MMP2 fibronectin}

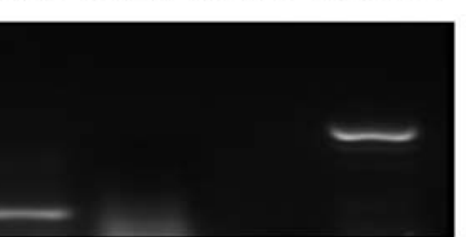

241

124

378

619

439

Figure 4. PCR analysis with $62.5 \mathrm{ng} / \mathrm{lane}$ of transcripts of the MSC markers CD73, CD90, CD105 and additionally matrix metalloproteinase 2 (MMP-2) and fibronectin was performed in MSC ${ }^{\mathrm{GFP}}$ compared to $\mathrm{MSC}^{\mathrm{GFP}}$-derived exosomes. The length of the PCR products was indicated in base pairs (bp).

A

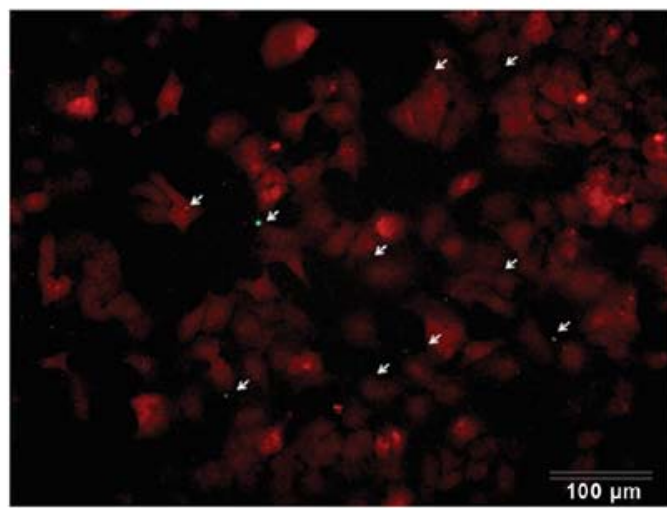

Incorporation of MSC ${ }^{\text {GFP-derived exosomes into }}$

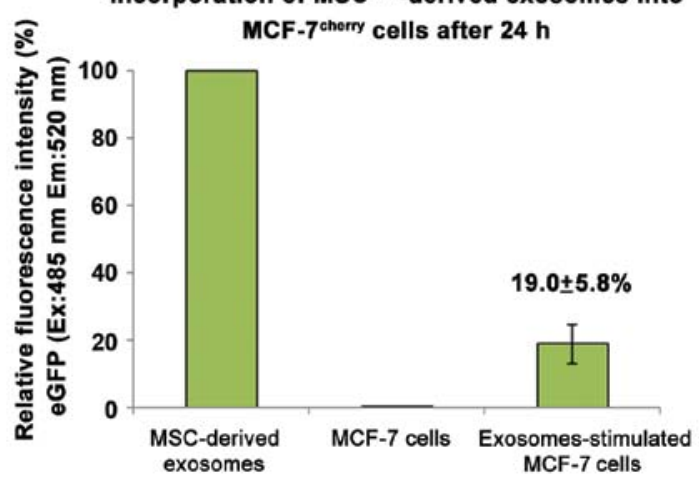

B

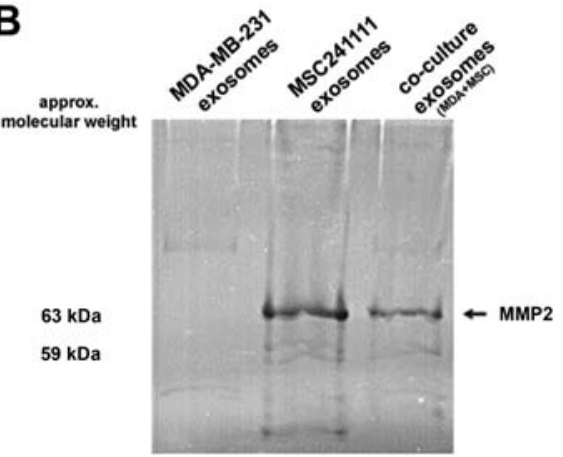

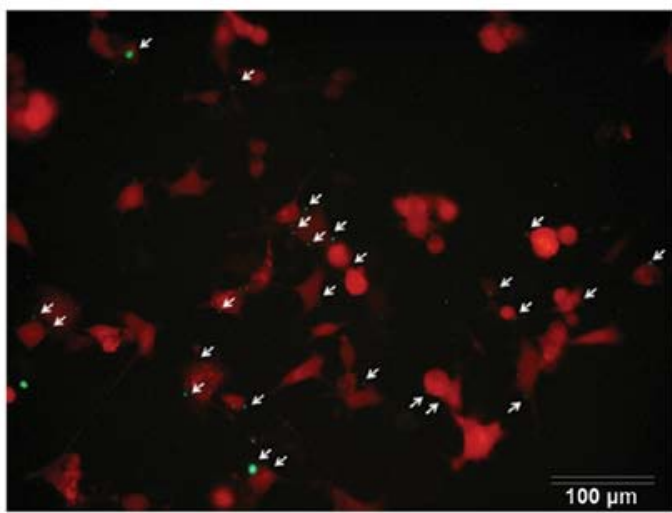

Incorporation of MSC ${ }^{\text {GFP }}$-derived exosomes into SCCOHT-1 $1^{\text {cherry }}$ cells after $24 \mathrm{~h}$

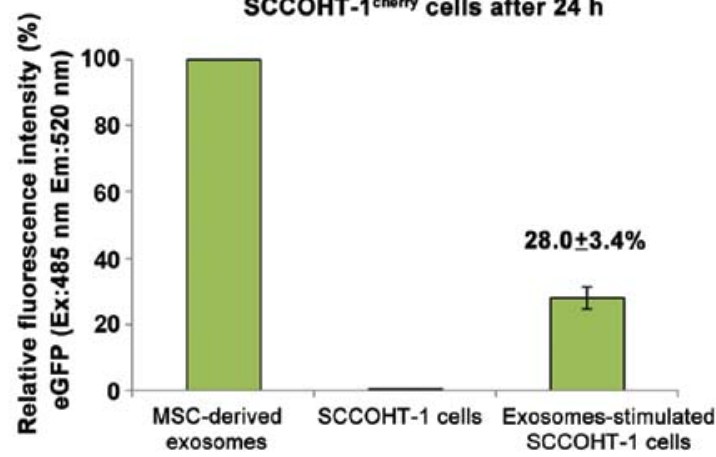

C

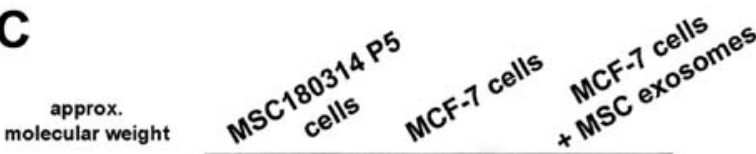

$63 \mathrm{kDa}$

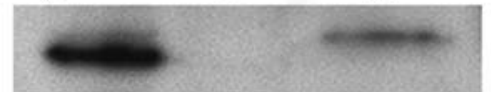

MMP2

$37 \mathrm{kDa}$

GAPDH

Figure 5. Incorporation and enzymatic analysis of exosomes. (A) Uptake of MSC ${ }^{\mathrm{GFP}}$-derived exosomes by MCF-7 ${ }^{\text {cherry }}$ breast cancer cells (upper left) or by

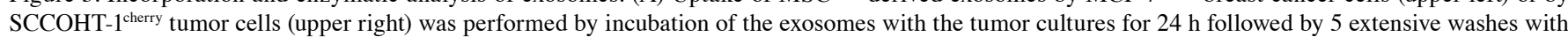
PBS to remove non-incorporated exosomes (bars represent $100 \mu \mathrm{m}$ ). The relative incorporation of MSC ${ }^{\mathrm{GFP}}$-derived exosomes into MCF-7 ${ }^{\text {cherry }}$ cells (lower left panel) or into SCCOHT- $1^{\text {cherry }}$ cells (lower right panel) within $24 \mathrm{~h}$ of incubation was quantified by a fluorescence-based assay whereby the fluorescence of GFP-labeled exosomes used for the cell stimulation was calculated as $100 \%$. Data represent the mean \pm SD (n=3). (B) Zymography of MMP-2 for gelatinase activity was determined with $5.8 \mu \mathrm{g}$ of exosomal protein from MDA-MB-231 and MSC mono-cultures and from 8-day co-culture of MSC/breast cancer cells, respectively. (C) MMP-2 western blot analysis of MSC and MCF-7 cell cultures compared to MCF-7 cells after incorporation of MSC-derived exosomes was performed by analysis of $40 \mu \mathrm{g}$ protein cell lysate/lane. Expression levels of GAPDH were used as a loading control. 

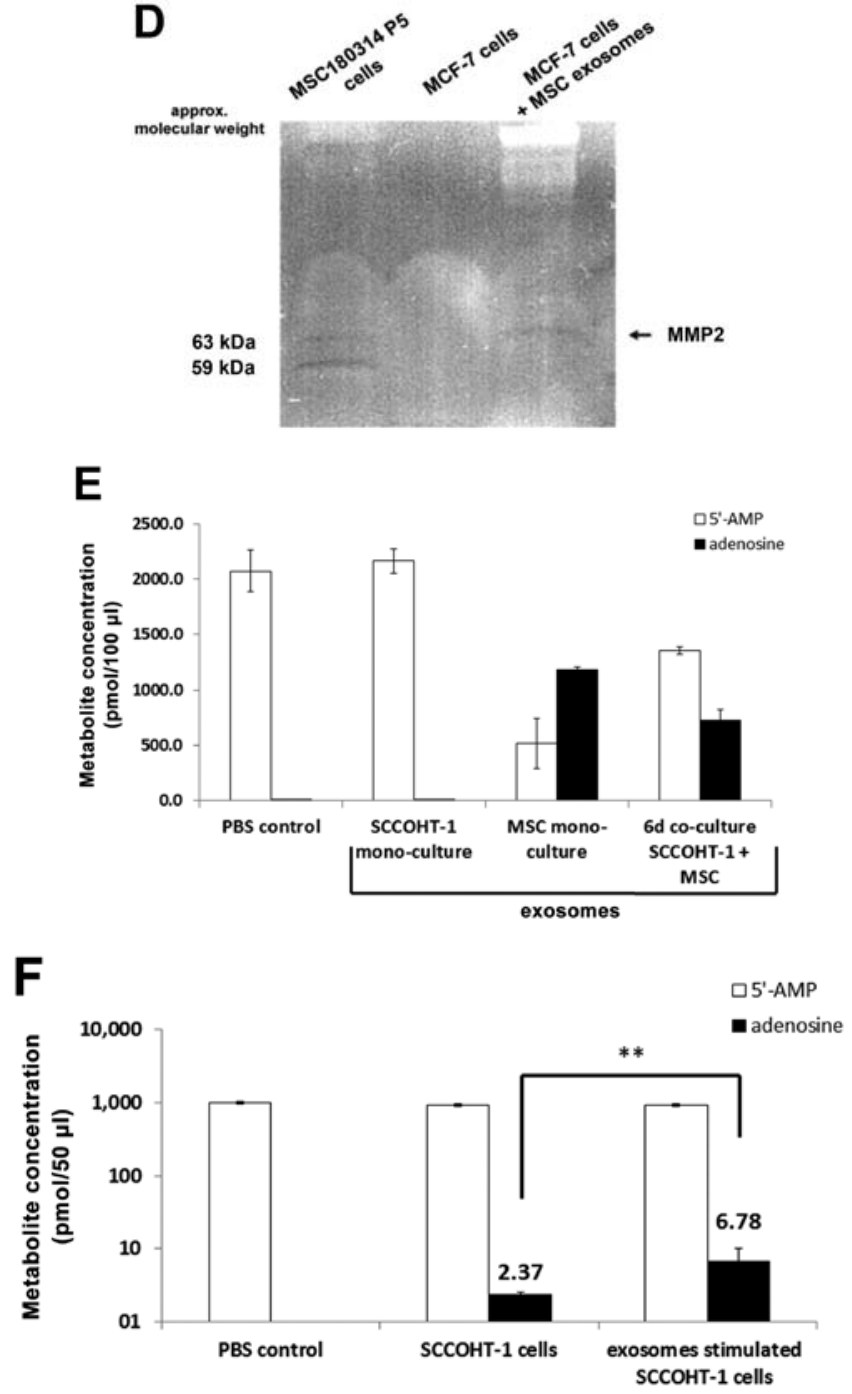

Figure 5. Continued. (D) Detection of MMP-2 gelatinase activity by a zymography assay of MSC and MCF-7 cell cultures compared to MCF-7 cells after incorporation of MSC-derived exosomes. (E) Ecto-5'-nucleotidase (CD73) activity was determined by the capacity of exosomes from equal cell amounts of SCCOHT-1 and MSC mono-cultures and from 6-day coculture of MSC/SCCOHT-1 cells to metabolize 5'-AMP into adenosine. The metabolites were analyzed by liquid chromatography combined with tandem mass spectrometry. Data represent the mean \pm SD of 3 experiments. (F) Ecto-5'-nucleotidase (CD73) activity was determined in SCCOHT-1 cells compared to SCCOHT-1 cells after 24-h incubation in the presence of MSCderived exosomes. Quantification of 5'-AMP and adenosine was performed by HPLC-MS/MS. Data represent the mean \pm SD of 3 experiments.

appeared in MSC-derived exosomes although in reduced quantities as compared to the cells. However, PCR products of CD105 and MMP-2 remained undetectable in these vesicles (Fig. 4) suggesting that only the proteins are carried.

Incubation of $\mathrm{MSC}^{\mathrm{GFP}}$-derived exosomes with tumor cells was associated with an uptake of exosomes by MCF-7 $7^{\text {cherry }}$ cells (Fig. 5A, upper left) and by SCCOHT-1 ${ }^{\text {cherry }}$ cells (Fig. 5A, upper right). The tumor cell cultures were incubated with the exosomes for $24 \mathrm{~h}$ and analysis of incorporated exosomes was performed after 5 extensive washes with PBS to remove free and loosely cell-attached exosomes. Quantification of incorporated exosomes into the cells was performed by fluorescence measurement of the GFP-labeled vesicles and revealed a relative uptake of $19.0 \pm 5.8 \%(n=3)$ by MCF-7 cells (Fig. 5A, lower left) and 28.0 $\pm 3.4 \%(n=3)$ by SCCOHT-1 cells (Fig. 5A, lower right) after $24 \mathrm{~h}$ compared to the total amount of available exosomes.

To test, whether the assimilation of MSC-derived exosomes by tumor cells includes transfer of biologically active proteins, gelatinase activity was measured for MMP-2 in an appropriate gelatin zymography assay. In contrast to exosomes from MDA-MB-231, MSC-derived exosomes and exosomes isolated from the MSC/breast cancer cell co-culture exhibited a marked gelatinase activity at $\sim 63 \mathrm{kDa}$, which corresponded to the active form of MMP-2 (Fig. 5B). A potential MSC-mediated transfer of MMP-2 was also tested for MCF-7 breast cancer cells. MSC cell homogenates demonstrated MMP-2 protein expression in contrast to undetectable MMP-2 levels in MCF-7 cells. However, incubation of MCF-7 cells in the presence of MSC-derived exosomes and subsequent removal of non-incorporated exosomes by extensive washes of the cells was associated with MMP-2 protein expression in the breast cancer cells (Fig. 5C). Moreover, the acquired MMP-2 expression in MCF-7 cells by MSC-derived exosomes was also tested for enzymatic activity. Whereas MSC cell homogenates exhibited MMP-2 gelatinase in-gel activity at $~ 59$ and $63 \mathrm{kDa}$, there was little if any MMP-2 activity detectable in MCF-7 cell homogenates. In contrast, MMP-2 activity was displayed in MCF-7 cells after incorporation of MSC-derived exosomes in the zymography assay (Fig. 5D).

Further acquisition of enzymatic activity from MSC-derived exosomes was evaluated for the CD73 ecto5 '-nucleotidase which associates to the external face of the plasma membrane via a GPI-anchor. The exosomal transfer of biologically active CD73 was tested by the capability to metabolize 5'-AMP into adenosine using a tumor cell culture model of primary cells from a small cell hypercalcemic ovarian carcinoma (SCCOHT-1). The preparation of exosomes from SCCOHT-1 cells demonstrated no detectable adenosine synthesis similar to PBS control incubation without any cells (Fig. 5E). In contrast, LC/MS-MS analysis of exosomes from MSC mono-culture and from MSC/SCCOHT-1 co-culture revealed a marked reduction of the substrate 5'-AMP paralleled by a significantly increased level of the product adenosine (Fig. 5E) suggesting CD73 activity exclusively in MSC-derived exosomes. Transfer of CD73 became obvious when a markedly elevated ecto-5'-nucleotidase activity was detectable after incorporation of MSC-derived exosomes into SCCOHT-1 cells compared to SCCOHT-1 control cells (Fig. 5F).

\section{Discussion}

Previous research has demonstrated that mesenchymal stem/stroma cells contribute to a direct interaction with tumor cells and promote mutual exchange/induction of cellular markers $(14,16,17)$. Alternatively, MSC interaction can be mediated indirectly by the release of soluble biological factors (18) and/or vesicles such as exosomes whereby MSC can affect cellular functionality of distant cell populations in a paracrine manner. These effects can be mediated both, by proteins and RNAs including mRNAs and miRs.

The appearance of the MSC marker proteins CD73, CD90 and CD105 in MSC-derived exosomes has been confirmed by 
previous studies (19). In addition, MSC-derived exosomes also contain transcripts for CD73, CD90, and fibronectin, whereas CD105 expression remained undetectable. Likewise, MMP-2 mRNAs were not observed in MSC-derived exosomes, suggesting appropriate protein transport in the microvesicles or regulators that induced expression in the target cells. Previous research has demonstrated that cancer cell-associated fibronectin induces release of matrix metalloproteinase- 2 from normal fibroblasts (20). Consequently, MMP-2 protein appearance in MCF-7 and MDA-MB-231 breast cancer cell populations with corresponding enzymatic activity after incorporation of MSC-derived exosomes enables degradation of certain collagens as structural component of basement membranes. Therefore, the acquisition of distinct matrix metalloproteinase activities suggested new properties of the tumor cells with the capability to restructure the tumor microenvironment. Indeed, transfection of MDA-MB-231 human breast cancer cells with pro-matrix metalloproteinase- 2 increased growth and metastasis in nude mice (21). Moreover, supportive evidence demonstrated that human adipose MSC-derived exosomes in conditioned medium promote migratory activity of MCF-7 cells accompanied by an upregulation of several cancer-related pathways such as Wnt signaling (22).

The incorporation of MSC-derived exosomes was also associated with acquired ecto-5'-nucleotidase activity by SCCOHT-1 tumor cells whereby this interaction included both, protein and corresponding mRNA assimilation. Previous findings substantiated acquisition of 5'-nucleotidase activity by SCCOHT-1 cells (17) and by normal natural killer cells (23) following direct co-culture with MSC which suggested a transfer by the cells and/or by the exosomes. With this new capability of metabolizing 5'-AMP into adenosine, SCCOHT-1 cells can suppress and modulate pro-inflammatory activities via activation of adenosine receptor signaling present on the surface of most immune cells (24). Indeed, previous reports demonstrated that exosomal conversion of 5'-AMP to adenosine can inhibit $\mathrm{T}$ cell activation in a tumor microenvironment (25).

Together, these findings suggested that MSC-derived exosomes can change the cellular functionality of tumor cells by induction of MMP-2 and ecto-5'-nucleotidase activity and thereby contribute to an altered tumor microenvironment and increased tumor heterogeneity $(26,27)$. Further factors including certain microRNAs in MSC-derived exosomes can also hide metastatic breast cancer cells by inducing dormancy (28). Thus, functional changes by MSC-derived exosomes can support a protection of tumor cells against chemotherapeutic approaches and consequently promote tumor cell resistance. Alternatively, MSC-derived exosomes may represent a useful carrier to deliver antitumor cargo.

\section{Acknowledgements}

Yuanyuan Yang is a visiting research fellow from Tongji University, Shanghai, China.

\section{References}

1. Caplan AI: Mesenchymal stem cells. J Orthop Res 9: 641-650, 1991.
2. Pittenger MF, Mackay AM, Beck SC, Jaiswal RK, Douglas R, Mosca JD, Moorman MA, Simonetti DW, Craig S and Marshak DR: Multilineage potential of adult human mesenchymal stem cells. Science 284: 143-147, 1999.

3. Majore I, Moretti P, Hass R and Kasper C: Identification of subpopulations in mesenchymal stem cell-like cultures from human umbilical cord. Cell Commun Signal 7: 6, 2009.

4. Dominici M, Le Blanc K, Mueller I, Slaper-Cortenbach I, Marini F, Krause D, Deans R, Keating A, Prockop DJ and Horwitz E: Minimal criteria for defining multipotent mesenchymal stromal cells. The International Society for Cellular Therapy position statement. Cytotherapy 8: 315-317, 2006.

5. Théry C, Regnault A, Garin J, Wolfers J, Zitvogel L, Ricciardi-Castagnoli P, Raposo G and Amigorena S: Molecular characterization of dendritic cell-derived exosomes. Selective accumulation of the heat shock protein hsc73. J Cell Biol 147: 599-610, 1999.

6. Valadi H, Ekström K, Bossios A, Sjöstrand M, Lee JJ and Lötvall JO: Exosome-mediated transfer of mRNAs and microRNAs is a novel mechanism of genetic exchange between cells. Nat Cell Biol 9: 654-659, 2007.

7. Lai RC, Tan SS, Teh BJ, Sze SK, Arslan F, de Kleijn DP, Choo A and Lim SK: Proteolytic potential of the MSC exosome proteome: Implications for an exosome-mediated delivery of therapeutic proteasome. Int J Proteomics 2012: 971907, 2012.

8. Yu B, Zhang X and Li X: Exosomes derived from mesenchymal stem cells. Int J Mol Sci 15: 4142-4157, 2014.

9. Lee JK, Park SR, Jung BK, Jeon YK, Lee YS, Kim MK, Kim YG Jang JY and Kim CW: Exosomes derived from mesenchymal stem cells suppress angiogenesis by down-regulating VEGF expression in breast cancer cells. PLoS One 8: e84256, 2013.

10. Zhou Y, Xu H, Xu W, Wang B, Wu H, Tao Y, Zhang B, Wang M, Mao F, Yan Y, et al: Exosomes released by human umbilical cord mesenchymal stem cells protect against cisplatin-induced renal oxidative stress and apoptosis in vivo and in vitro. Stem Cell Res Ther 4: 34, 2013.

11. Zhu W, Huang L, Li Y, Zhang X, Gu J, Yan Y, Xu X, Wang M, Qian $\mathrm{H}$ and $\mathrm{Xu}$ W: Exosomes derived from human bone marrow mesenchymal stem cells promote tumor growth in vivo. Cancer Lett 315: 28-37, 2012.

12. Lavrentieva A, Majore I, Kasper C and Hass R: Effects of hypoxic culture conditions on umbilical cord-derived human mesenchymal stem cells. Cell Commun Signal 8: 18, 2010.

13. Otte A, Göhring G, Steinemann D, Schlegelberger B, Groos S, Länger F, Kreipe HH, Schambach A, Neumann T, Hillemanns P, et al: A tumor-derived population (SCCOHT-1) as cellular model for a small cell ovarian carcinoma of the hypercalcemic type. Int J Oncol 41: 765-775, 2012.

14. Mandel K, Yang Y, Schambach A, Glage S, Otte A and Hass R: Mesenchymal stem cells directly interact with breast cancer cells and promote tumor cell growth in vitro and in vivo. Stem Cells Dev 22: 3114-3127, 2013.

15. Otte A, Bucan V, Reimers K and Hass R: Mesenchymal stem cells maintain long-term in vitro stemness during explant culture. Tissue Eng Part C Methods 19: 937-948, 2013.

16. Hass R and Otte A: Mesenchymal stem cells as all-round supporters in a normal and neoplastic microenvironment. Cell Commun Signal 10: 26, 2012.

17. Yang Y, Otte A and Hass R: Human mesenchymal stroma/stem cells exchange membrane proteins and alter functionality during interaction with different tumor cell lines. Stem Cells Dev: Jan 26, 2015 (Epub ahead of print).

18. Karnoub AE, Dash AB, Vo AP, Sullivan A, Brooks MW, Bell GW, Richardson AL, Polyak K, Tubo R and Weinberg RA: Mesenchymal stem cells within tumour stroma promote breast cancer metastasis. Nature 449: 557-563, 2007.

19. Kim HS, Choi DY, Yun SJ, Choi SM, Kang JW, Jung JW, Hwang D, Kim KP and Kim DW: Proteomic analysis of microvesicles derived from human mesenchymal stem cells. J Proteome Res 11: 839-849, 2012.

20. Saad S, Gottlieb DJ, Bradstock KF, Overall CM and Bendall LJ: Cancer cell-associated fibronectin induces release of matrix metalloproteinase-2 from normal fibroblasts. Cancer Res 62: 283-289, 2002

21. Tester AM, Waltham M, Oh SJ, Bae SN, Bills MM, Walker EC, Kern FG, Stetler-Stevenson WG, Lippman ME and Thompson EW: Pro-matrix metalloproteinase-2 transfection increases orthotopic primary growth and experimental metastasis of MDA-MB-231 human breast cancer cells in nude mice. Cancer Res 64: 652-658, 2004. 
22. Lin R, Wang S and Zhao RC: Exosomes from human adiposederived mesenchymal stem cells promote migration through Wnt signaling pathway in a breast cancer cell model. Mol Cell Biochem 383: 13-20, 2013.

23. Chatterjee D, Tufa DM, Baehre H, Hass R, Schmidt RE and Jacobs R: Natural killer cells acquire CD73 expression upon exposure to mesenchymal stem cells. Blood 123: 594-595, 2014.

24. Ohta A and Sitkovsky M: Extracellular adenosine-mediated modulation of regulatory T cells. Front Immunol 5: 304, 2014.

25. Clayton A, Al-Taei S, Webber J, Mason MD and Tabi Z: Cancer exosomes express CD39 and CD73, which suppress T cells through adenosine production. J Immunol 187: 676-683, 2011.
26. Ungefroren H, Sebens S, Seidl D, Lehnert H and Hass R: Interaction of tumor cells with the microenvironment. Cell Commun Signal 9: 18, 2011.

27. Friedl $\mathrm{P}$ and Alexander S: Cancer invasion and the microenvironment: Plasticity and reciprocity. Cell 147: 992-1009, 2011.

28. Ono M, Kosaka N, Tominaga N, Yoshioka Y, Takeshita F, Takahashi RU, Yoshida M, Tsuda H, Tamura K and Ochiya T: Exosomes from bone marrow mesenchymal stem cells contain a microRNA that promotes dormancy in metastatic breast cancer cells. Sci Signal 7: ra63, 2014. 